\title{
A Novel Approach and Application of Time Series to Image Transformation Methods on Classification of Underwater Objects
}

\author{
Aybuke CIVRIZOGLU BUZ ${ }^{*}$ a $\left(\mathbb{D}\right.$, Mustafa Umut DEMIREZEN ${ }^{\mathrm{b}}$, (D),Uraz YAVANOGLU ${ }^{\mathrm{c}}$, \\ ${ }_{a, *}{ }^{*}$ HAVELSAN Command Control and Defense Technologies,06510,Ankara, Turkey. \\ ${ }^{b}$ ROKETSAN Artificial Intelligence and Technology Management, 06780, Ankara, Turkey. \\ ${ }^{c}$ Gazi University, Faculty of Engineering,Department of Computer Engineering, 06570, Ankara, Turkey.
}

\section{ARTICLE \\ INFO \\ Received: 15.01 .2021 \\ Accepted: 04.04.2021 \\ Keywords: \\ MTF, GAF, RP, \\ Sonar, Deep Learning}

\begin{abstract}
Sonar is used to determine the size, distance, direction, and other object features using sound waves. It is widely used in submarine mining, oil exploration, submarine mapping, tracking fish shoals, and mine detection. In Machine Learning (ML) research, feature extraction, selection, algorithm selection, and hyper-parameter optimization, which should be used to identify and classify sonar signals, are seen as scientific problems studied for many years. In this study, instead of commonly used ML algorithms and feature extraction processes, three different mathematical transformations were suggested to classify the underwater objects as an innovative approach. This novel approach applied on a data set in time-series format, data has been transformed from onedimensional data to a two-dimensional format and a simple channel merging technique was applied to create a new image joining these images. The methods' performance was measured by the classification results of mines and rocks using deep learning algorithms on the sonar dataset. Moreover, the performance results obtained with deep learning, compared with the classical algorithms. Finally, confronted with other studies in the literature, it has been seen that the proposed time-series data-to-image transformation with a channel-merging approach eliminates the need for feature extraction and achieves superior results against the others.
\end{abstract}

\section{Sualtı Nesnelerinin Sınıflandırılmasında Zaman Serisi Görüntü Dönüşümü Yöntemlerinin Yeni Bir Yaklaşımı ve Uygulaması}

\section{MAKALE BİLGISİ \\ Alınma: 15.01.2021 \\ Kabul: 04.04.2021 \\ Anahtar Kelimeler: MTF, GAF, RP, \\ Sonar, Derin Öğrenme}

\section{öz}

Sonar, ses dalgalarını kullanarak boyutu, uzaklığı, yönü ve diğer nesne özelliklerini belirlemek için kullanılır. Denizaltı madenciliği, petrol arama, denizaltı haritalama, balık sürülerinin takibi ve mayın tespitinde yaygın olarak kullanılmaktadır. Makine Öğrenimi araştırmasında, sonar sinyallerini tanımlamak ve sınıflandırmak için kullanılması gereken özellik çıkarma, seçme, algoritma seçimi ve hiper parametre optimizasyonu, uzun yıllardır çalışılan bilimsel problemler olarak görülmektedir. Bu çalışmada, yaygın olarak kullanılan makine öğrenimi algoritmaları ve öznitelik çıkarma süreçleri yerine, su altı nesnelerini yenilikçi bir yaklaşım olarak sınıflandırmak için üç farklı matematiksel dönüşüm önerilmiştir. Zaman serisi formatında bir veri setine uygulanan bu yeni yaklaşım, veriler tek boyutlu verilerden iki boyutlu bir formata dönüştürülmüş ve bu görüntüleri birleştiren yeni bir görüntü oluşturmak için basit bir kanal birleştirme tekniği uygulanmıştır. Yöntemlerin performansı, sonar veri setinde derin öğrenme algoritmaları kullanılarak maden ve kayaların sınıflandırma sonuçlarıyla ölçülmüştür. Ayrıca klasik algoritmalara göre derin öğrenme ile elde edilen performans sonuçları. Son olarak, literatürdeki diğer çalışmalarla karşılaşıldığında, önerilen zaman serisi verilerden görüntüye dönüşümün kanal 
birleştirme yaklaşımı ile öznitelik çıkarma ihtiyacını ortadan kaldırdığı ve diğerlerine göre üstün sonuçlar elde ettiği görülmüştür.

https://dx.doi.org/10.30855/gmbd.2021.01.01

\section{INTRODUCTION (GIRIŞs)}

In recent years, exploration of the underwater world, detection and classification of underwater objects and targets occupy an essential place in many applications for civil and military purposes. Sonar (Sound Navigation and Ranging), which is used to explore the underwater world, is used to gain information about the size, distance, and structure of objects using sound waves [1]. Although sonar systems were initially developed for naval warfare, today they are used in many essential areas with the purpose of oil and mineral exploration, seafloor mapping, detection of fish shoals, measurement of waves and currents, detection of sunken ships, drowned and submerged people, wreckage such as aircraft and underwater search and rescue purposes in many essential areas [2]. Today, sonar systems occupy a vital place in the defense industry for underwater target and mine detection [3]. Not only in times of war, but especially in times of peace, often used to protect coastal security, ship landings, and port controls, and such matters as conducting sonar systems healthily, have started to offer more advanced opportunities in the fields with the development of technology [4]. With the development of the technology, both the quality and the features of the signals, and images received from sonar devices have increased. Recently, although the lack of data sets and resources, especially for mines and similar objects, several studies conducted with both images and signals has made research in this field difficult, still there are a limited number of studies based on machine learning based and statistical analysis methods published in the literature [4], [5], [6], and [7].

Detection of mine objects with artificial intelligence algorithms becomes difficult due to the high diversity of underwater mines, the similarity of other objects such as rocks, debris, and cables to mines in shape and structure, signal reflections, and disruptive noise effects on the signal. Classical machine learning algorithms are based on feature extraction. Obtaining these attributes explicitly developed for the problem can be done with field expertise. Besides, choosing the most important features among the produced features plays a vital role in training machine learning algorithms and developing and improving the results. As a result of the increase in the number of features named "curse of dimension" [8], generating an excessive number of features causes an exponential increase in the number of training data required for the algorithm. Using less training data and too many attributes cause the algorithm to overfit. When such situations arise, machine learning algorithms get unsuccessful results in classification or regression problems because they lose their ability to generalize. Although deep learning methods, in which feature extraction and selection processes can be performed automatically instead of classical algorithms, provide more advantages in this regard, they require more data to achieve successful results.

This study proposes a new method for the binary classification problem using a dataset containing sonar signal data obtained using cylindrical mines and cylindrical rocks similar in shape and size to mines [9]. Using the proposed method, we focused on the classification problem of rocks and mines, a binary classification problem, and compared classical machine learning algorithms and deep learning algorithms. Compared to other literature studies, our earlier work on converting one-dimensional timeseries data to image format with an RGB channel was used [21]. Changing the data representation ensured that convolutional neural networks-based deep learning architectures were used, and it was determined that successful results were obtained with limited data. In addition to this, to leverage the information obtained from three different transformations, we proposed to add one more step by joining these transformation results as one image with three different image channels (channel merging). The aim is to exploit more information obtained from time series to image conversion with three different mathematical transforms and merge this information as an image channel to form a new image as a new representation. This new representation provides more information than single transformation methods and inherits specific advantages specific to underlying transformation.

In this paper; in the second section, a brief literature review summarizes the algorithms used and examines other studies using the dataset used in this paper. In the third section, the mathematical model and the technical explanation of the innovative approach used in this study are given. In the last section, all the results for experiments performed in 
order to compare algorithm performances are shown, and the findings are discussed.

\section{LITERATURE REVIEW (LITERATÜR ÖZETI)}

A study carried out with inside the area of classification of underwater objects [9] determines whether or not the target is a cylindrical mine or a cylindrical rock in line with the returning sign from two distinctive underwater objects. In this study, to examine the consequences of the range of hidden layers and the viewing angle at the result, experiments were made in distinctive hidden layers and relying on and not relying on the viewing angle. K-Nearest Neighbor Classification algorithm (K-NN) is used in their studies. The overall performance of the individuals who tested those signals with the latest consequences became in comparison, and it became observed that human overall performance remained low in comparison to the studies. The ensuing eminent accuracy value of 90.4 depends at the viewing angle and received in trials with 12 hidden layers. In any other study performed with the identical facts set [10], the Generalized Regression Neural Network (GRNN) method became used to categorize the sonar data set. While the usage of the GRNN method, once they added the function extraction method referred to as Principal Component Analysis (PCA) to the study, it was discovered that the class overall performance is ascended. In this study, the accuracy of the type was evaluated with the aid of using ROC analysis which is often used to indicate the reliability and generalization of data sets. The result was $91.34 \%$ whilst solely the GRNN method was used; while the result was $93.26 \%$ when PCA was used in addition to GRNN. In another study carried out in this problem [11], 9 sample-based genetic algorithms (GA) classifiers have been utilized in diverse versions to reach a higher overall performance in generalization capacity and training efficiency. The overall performance acquired due to those versions was compared. The 9 methods mentioned were examined with 19 datasets. The sonar dataset's maximum fulfillment rate was $53.43 \%$ with inside the version named TS2. In another study the usage of the C4.5 decision tree [12], the problem of classifying sonar targets with the aid of using enhancing the overall performance of the C4.5 decision tree with the aid of using the Knowledge Acquisition (IG) feature assessment process became studied. Another point well worth noting on this study is that feature selection improves computational efficiency while augmenting the classification accuracy. It has been stated that the accuracy of the C4.5 algorithm, which is working with $\mathrm{BK}$, has increased by more than $10 \%$ and reached $81 \%$. In another study carried out at the analysis of sonar signals [13], sonar signals will grow to be a data set of boosting size with an uninterrupted data float. The overall performance problems of this size were investigated. It was emphasized that data processing time need to be kept as brief as possible to detect data with a continuous flow such as sonar signals in realtime. In this study, a brand new strategy called iDSM$\mathrm{CA}$, which is designed to clean noisy data step by step with the usage of fast collision analysis, is presented as a solution to this problem. With this strategy, sonar classification tests were applied with six classification algorithms in total. In another study carried out on a similar problem [14], it is aimed to clear up the complexity of the decision boundary that algorithms need to locate with inside the classification field. In order to remedy this problem, the Incremental Maximum Gaussian Mixture Partition (IMGMP) algorithm is proposed for classification. This algorithm makes use of a divide and conquers strategy to calculate an appropriate decision boundary. KMeans algorithm became used to split the data into clusters. It was emphasized that the overall performance of IMGMP is tremendously dependent on the cluster size in establishing the decision boundary, and a result with an accuracy of $82.79 \%$ was acquired. In another study [15], a nouveau algorithm is proposed to solve this problem of the $\mathrm{K}$ Nearest Neighbor Classification Algorithm (KNN), which occurs due to the fact the calculation cost increases whilst the scale of the data set increase. While the KNN algorithm calculates the space between the training data and the test data, the MIMEKNN algorithm has been proposed, as a way to maximize the space between classes and the minimum in-class distance because of the complexity of this calculation as the data set grows. As a result of the studies, whilst the KNN algorithm presents an accuracy rate of $74.50 \%$, the accuracy rate of the MIME-KNN algorithm has been acquired as $82.20 \%$. The effects of these studies are given in Table I with inside the Results section.

\section{MATERIAL AND METHODS (YÖNTEM VE TEKNIKLER)}

The proposed method is based on converting time series data to three-channel (RGB) or single-channel (gray) lossless image format [21]. In this transformation, the spatial and temporal changes of data in each frequency band are handled differently, and transformed into a three-dimensional picture format by one-dimensional data transformation. After these transformations are applied, spatial and temporal properties of time series are preserved and 
information stored as regular images. Recurrence plot (RP) and Markov transition field (MTF) transformations are a count-based probabilistic type of mathematical transformation, but Gramian angular difference field (GADF) and Gramian angular summation field (GASF) methods are a transformation method that takes into account the temporal and spatial correlation.

In this newly-converted picture format, it is possible to create additional features that cannot be used or obtained in one dimension, and with this information representation results in increasing classification performance. When the time series data is converted into image representation, the classification problem can be solved using classical image processing methods on these pictures. In addition to this, to leverage the knowledge acquired from three different transformations, As an additional step, appending one more operation to previous work [21] by combining these transform results in one image with three separate image channels (channel merging). The intention is to utilize more knowledge gained from time series to image conversion with three different mathematical transforms and merge it as an image channel to create a final image as a new representation. This new representation provides more knowledge than single transformation methods and presents specific advantages particular to underlying transformation.

In Figure 1. all data preparation and other necessary stages of the proposed method are shown. In the first step, every available time-series data are transformed to a matrix format with three different mathematical methods, and then by using these matrixes, a new image is formed by joining these transformed data as R, G, and B channels of the data. Then training and test sets are sampled from the data set from images, and a Convolutional Neural Network model is trained with the K-Fold cross validation method. Finally, Performance metrics such as precision, recall, F1 - score, and accuracy values are calculated for the model.

Two hundred and eight different signals obtained from 60 different bands from the sonar device were broadcast in time-series format [9]. Data in this format is transformed into an image format (RGB) using transformations with specific properties. In the study, the visual transformation of the time series data was carried out with three different methods called Gramian Angular Field, Markov Transition Field, and Recurrence Plot.

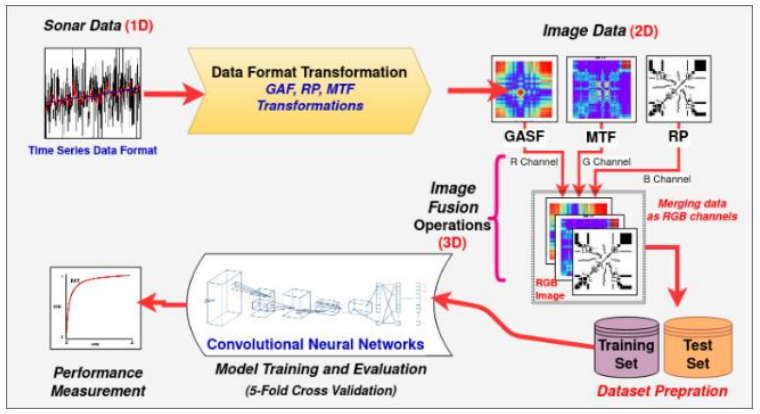

Figure 1. Flow chart for the proposed method (Önerilen yöntem için akış diyagramı)

\subsection{Time Series-Image Transformation Approach (Zaman Serisi Görüntü Dönüşümü Yaklaşımı)}

In this study, three different mathematical transformation methods are used to obtain images from the time series. These methods were used as Gramian Angular Field (GAF), Markov Transition Field (MTF) [16], [17], and Recurrence Plot (RP) [18], respectively. The RGB color map represents the values transformed by GAF and MTF methods. In the color map, colors close to blue and blue represent smaller values, while colors close to red and red represent the values with larger amplitudes. RP, on the other hand, is represented by a black and white image with a single channel. The time-series data properties are defined as 2-dimensional images with colors, dots, and lines at the corresponding positions in the image in which they are transformed and differ from each other.

\subsubsection{Gramian angular field transformation (Gramian açısal alan dönüşümü)}

In the angular field, the time series is represented in the polar coordinate plane instead of the Cartesian coordinate plane. In the GAF, each element is the cosine of the sum of the angles. GAF has two different methods. These methods are Gramian Angular Summation Field / GASF Gramian Angular Difference Field / GADF. In the Gramian angular area method, each point in the given time series is rescaled. So each value in Eq. (1) with [-1, 1] or in Eq. (2) with $[0,1]$.

$$
\begin{aligned}
& \tilde{x}_{-1}^{i}=\frac{\left(x_{i}-\max (X)+x_{i}-\min (X)\right)}{\max (X)-\min (X)} \\
& \tilde{x}_{0}^{i}=\frac{x_{i}-\min (X)}{\max (X)-\min (X)}
\end{aligned}
$$

The obtained new time series values are expressed in the polar coordinate plane by encoding the time stamp as angular cosine, and the time stamp as a radius. In Eq. (3). Here $\varnothing$ angle is a fixed parameter for regulating the polar coordinate, $t_{i}$ timestamp, $\mathrm{r}$ radius, and $\mathrm{N}$ polar coordinate plane. 


$$
\begin{aligned}
& \left\{\begin{array}{c}
\emptyset=\arccos \left(\tilde{x}_{i}\right),-1 \leq \tilde{x}_{i} \leq 1, \tilde{x}_{i} \in \tilde{X} \\
r=\frac{t_{i}}{N}, t_{i} \in \mathbb{N}
\end{array}\right. \\
& G A S F=\left[\begin{array}{ccc}
\cos \left(\emptyset_{1}+\emptyset_{1}\right) & \cdots & \cos \left(\emptyset_{1}+\emptyset_{n}\right) \\
\vdots & \ddots & \vdots \\
\cos \left(\emptyset_{n}+\emptyset_{1}\right) & \cdots & \cos \left(\emptyset_{n}+\emptyset_{n}\right)
\end{array}\right] \\
& G A S F=\tilde{X}^{\prime} \cdot \tilde{X}-{\sqrt{I-\tilde{X}^{2}}}^{\prime} \cdot \sqrt{I-\tilde{X}^{2}}
\end{aligned}
$$

After the time series are rescaled, they are transformed into a polar coordinate system with the help of Eq. (3). Using this data, converted into a polar coordinate system, according to the desired output, trigonometric addition or difference is transformed into matrix structure via Gramian Angular Summation Field (GASF) Eq. (4), Eq. (5) or Gramian Angular Difference Field (GADF) Eq. (6), Eq. (7). The conversion is completed by using the cosine functions for GASF and the sine functions for GADF.

$$
\begin{gathered}
G A D F=\left[\begin{array}{ccc}
\sin \left(\emptyset_{1}-\emptyset_{1}\right) & \cdots & \sin \left(\emptyset_{1}-\emptyset_{n}\right) \\
\vdots & \ddots & \vdots \\
\sin \left(\emptyset_{n}-\emptyset_{1}\right) & \cdots & \sin \left(\emptyset_{n}-\emptyset_{n}\right)
\end{array}\right] \\
G A D F=\sqrt{I-\tilde{X}^{2}} \cdot \tilde{X}-\tilde{X}^{\prime} \cdot \sqrt{I-\tilde{X}^{2}}
\end{gathered}
$$

At the end of this step, the data in the time series format is encoded in a picture format containing 3channel RGB color coding, and the transformation process is completed. An application of the described method is shown in Figure 2.

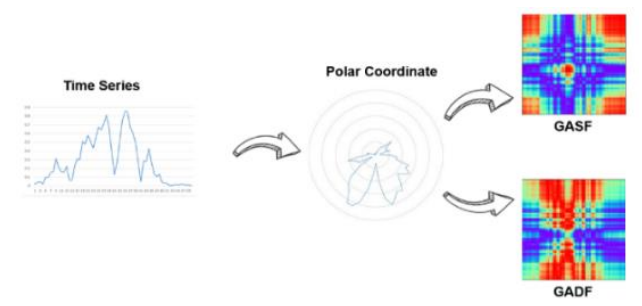

Figure 2: Gramian angular field transformation (Gramian açısal alan dönüşümü)

\subsubsection{Markov transition field transformation (Markov geçiş alanı dönüşümü)}

Markov transition matrix with transition probabilities for the time series decomposed in MTF is created. This matrix is then transformed into an image. In the Markov Transition Field, a matrix of size $Q \times Q$ is created from a given time series. First of all, the partitioning process is completed by defining the time series' quantitative divisions and assigning each to its corresponding quantile divisions. In the equation given in Eq. (8), it shows the Markov Transition
Matrix obtained by dividing the data by the Q quantile interval:

$$
M=\begin{array}{ccc}
w_{i j \mid x_{1} \in q_{i}, x_{1} \in q_{j}} & \cdots & w_{i j \mid x_{1} \in q_{i}, x_{n} \in q_{j}} \\
w_{i j \mid x_{2} \in q_{i}, x_{1} \in q_{j}} & \cdots & w_{i j \mid x_{2} \in q_{i}, x_{n} \in q_{j}} \\
\vdots & \ddots & \vdots \\
\left\lfloor w_{i j \mid x_{n} \in q_{i}, x_{1} \in q_{j}}\right. & \cdots & \left.w_{i j \mid x_{n} \in q_{i}, x_{n} \in q_{j}}\right\rfloor
\end{array}
$$

The transformation process is completed by encoding the probability-based matrix obtained with this transformation in a picture format that includes 3channel RGB color coding. An application of the method described is shown in Figure 3.

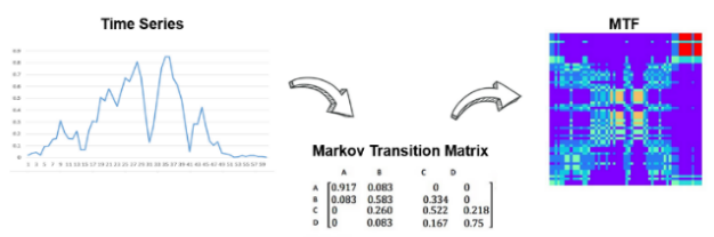

Figure 3: Markov transition field transformation (Markov geçiş alanı dönüşümü)

\subsubsection{Recurrence plot transformation (Tekrarlanma grafiği dönüşümü)}

Recurrence Plot; it is a method used for repetitive processes such as time series, periodic data, and cycles and visualizes these processes in two dimensions. RP is also an advanced technique that is frequently used in the applications of nonlinear data analysis. The primary purpose of recurrence plots is to examine the higher dimensional phase space trajectories visually. RP's can be applied to short and unstable data. In the Recurrence Plot method, the image is created by calculating the distance of each point in the time series to the same time series. In this method, which works to visualize a time-dependent behavior, the primary step of the visualization is calculating the $N \times N$ dimensional matrix obtained from the time series [18], a tool that can visualize the repetition of $x_{i}$ in $i^{\text {th }}$ states in phase space. The mathematical representation of the repetition graph is shown in Eq. (9).

$$
\mathbf{R}_{i, j}=\Theta\left(\varepsilon-\left\|\vec{x}_{i}-\vec{x}_{j}\right\|\right), i, j=1 \ldots N
$$

In Eq. (13) $\mathrm{E}$ is a threshold, $\mathrm{N}$ is the number of data points to be calculated, $\Theta$ is a stepwise function. This tool allows to investigate the m-dimensional phase space trajectory through a two-dimensional representation of its repetitions. Such a repetition of a state at a different time is marked by ones and zeros (black and white points on the graph) within the twodimensional square matrix. This representation is called the Recurrence Plot (RP). The transformation process is completed by encoding the bivalent matrix obtained in a picture format containing one channel 
color coding. An application of the method described is shown in Figure 4.
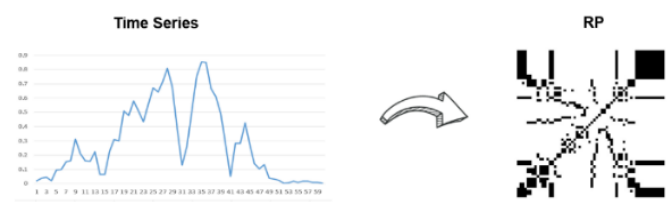

Figure 4. Recurrence plot transformation (Tekrarlanma grafiği dönüşümü)

\subsection{Image Data Merging (Dönüştürülmüsş Görüntü Verisinin Birleştirilmesi)}

After each transformation, as mentioned earlier, was applied to time-series data, three different results were obtained in a matrix format specific to that transformation method. $\boldsymbol{X}_{M T F}, \boldsymbol{X}_{G A F}$ and $\boldsymbol{X}_{R P}$ are resulting $\mathrm{N}$ by $\mathrm{N}$ dimensional matrixes $\left(X_{M T F}, X_{G A F}, X_{R P} \in \mathbb{R}^{N x N}\right)$ and $\boldsymbol{X}_{\text {Merged }}$ is the N-by-N $\mathrm{X} 3$ tensor $\left(X_{\text {Merged }} \in \mathbb{R}^{N x N x 3}\right)$. To leverage more information from different transformations, these matrixes can be merged to form a new matrix that can be interpreted as an RGB image.

$$
\begin{aligned}
& X_{\text {Merged }}^{i, j, 1}=X_{M T F}^{i, j} \\
& X_{\text {Merged }}^{i, j, 2}=X_{G A F}^{i, j} \\
& X_{\text {Merged }}^{i, j, 3}=X_{R P}^{i, j}
\end{aligned}
$$

In Eq 10-12, $\mathrm{i}, \mathrm{j} \in \mathrm{Z}$, are row and column indexes of the matrix and tensor respectively. Now, $\mathrm{X}_{\text {Merged }}$ represents all available information obtained from different transformations and as a result it forms an RBG image. This operation is applied all the time series data in the dataset and finally a new data set is formed completely in image format.

\section{DATASET (VERISETI)}

The data set [9] used in this study was created with the signals reflected from cylindrical mines and cylindrical rocks resembling these mines from different angles. The cylindrical mines and cylindrical rocks from which the sonar data sets were obtained are approximately $5 \mathrm{ft}$. long. There are a total of 208 data in the data set. These data were obtained by taking an average of 5 turns to be chosen randomly from each viewpoint out of 1200 signals sent as a return to signals sent from different angles, provided that they are up to 180 degrees for rocks and 90 degrees for mines. Of the 208 data obtained, 111 belong to cylindrical mines and 97 to cylindrical rocks. Each data is a set of 60 numbers, ranging from 0.0 to 1.0. Each number represents the energy collected over a fixed period in a given frequency band. Although each number does not precisely reflect the angle value, they are ordered in ascending angles. In the classification of each data, the data rock is labeled as $\mathrm{R}$, and the mine as M. In Figure 5 and Figure 6, the graph of the sonar signal returns in the time series axis belonging to all mines and all rocks in the data set.

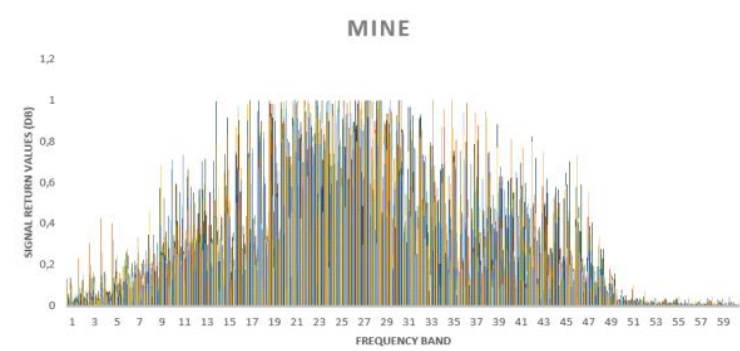

Figure 5. Signal return values corresponding to the frequency band measured for all mine targets (Tüm mayın hedefleri için ölçüm yapılan frekans bandına karşıllk sinyal dönüşü değerleri)

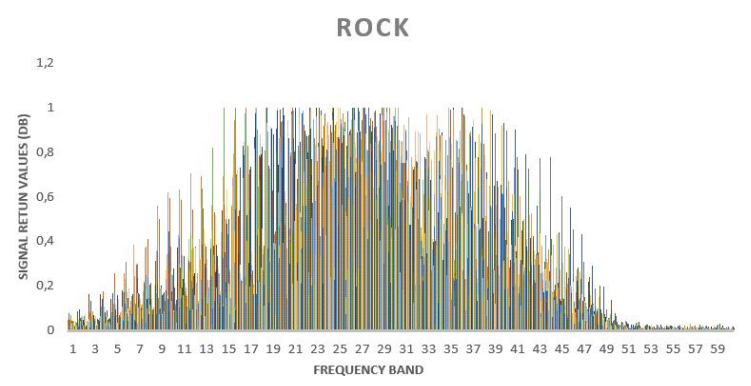

Figure 6. Signal return values corresponding to the frequency band measured for all rock targets (Tüm kaya hedefleri için ölçüm yapılan frekans bandına karşılık sinyal dönüşü değerleri)

\section{BRIEF DESCRIPTION OF USED MACHINE LEARNING METHODS (KULLANILAN MAKINE ÖĞRENMESİ ALGORITMALARININ KISA AÇIKLAMALARI)}

In this study, classical machine learning algorithms, which are frequently encountered in the literature, were selected for this binary classification problem. Selected classic machine learning algorithms are Support Vector Machine, Decision Tree Algorithm, K-Nearest Neighbor Algorithm, Linear Discriminant Analysis, Gauss NB, Logistic Regression, and Random Forest. Their performance was optimized by hyper-parameter selection specific to the algorithm. In the following subsections, these methods are briefly described and compared: 


\subsection{Convolutional Neural Networks (Evrişimli Sinir A}

Convolutional neural network is a deep neural network consisting of neurons with learnable weights that can process data strings such as image classification, widely used in computer vision. Convolutional Neural Networks (CNN) are a type of multilayer perceptron. CNN algorithms image and sound processing area natural language processing (NLP), especially in many different fields such as biomedical is implemented. Especially in the field of image processing achieve the best (state of the art) results seen have been. [19] As the proposed deep learning architecture, a custom convolutional neural network given in Figure 7 has been designed, and used due to the small number of data. It has been determined that a less complicated network is appropriate because other architectures commonly used in the literature generally overfit and memorize the data.

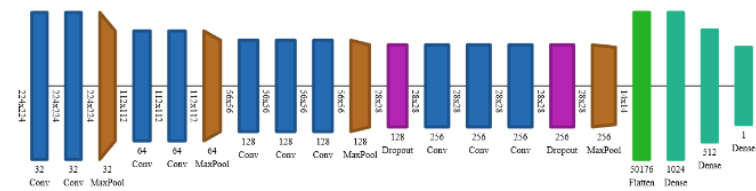

Figure 7. The convolutional neural network architecture used (Kullanılan evrişimli sinir ağı mimarisi)

\subsection{Support Vector Machines Algorithm (Destek Vektör Makineleri Algoritmast)}

SVM is a supervised learning algorithm, is a vector space-based algorithm that determines hyperplanes to separate data from each other by finding a decision boundary between data. Among the advantages of the SVM algorithm are that it is effective in high-dimensional data, makes memory usage more efficient thanks to the training points it uses, and makes versatile decisions with different kernel functions. SVM uses kernel methods to deal with nonlinear data because these methods create nonlinear combinations of the original properties of the data and make a mapping that separates these methods linearly and thanks to this mapping, a linear support vector machine algorithm transforms the training data into a higher dimensional feature area makes it trainable with. While performing all these operations, it uses kernel tricks since calculation cost will increase in cases where the size of the data set is too high.

\subsection{Decision Tree Algoritm (Karar Ağacı Algoritmast)}

DT algorithms are used as a solution to both classification, and regression problems. It is a tree structure consisting of decision trees, decision nodes, and leaf nodes. Using the decision algorithm, it is started from the tree root, and the data on the most informative features are divided and the process is repeated until the node takes its purest form. Decision trees can be a tree with many nodes with this method, resulting in over-sleeping. In order to prevent overfitting in decision tree algorithms, a limit should be determined for the maximum number of nodes of the tree to be formed, and pruning should be done. The decision tree algorithm can be used to process both numerical, and categorical data, and it can process high-dimensional data in a short time due to its low computational cost. For this reason, it is preferred when data size increases among alternative algorithms.

\subsection{K-Nearest Neighbor Algorithm (K-En Yakın Komşu Algoritması)}

$\mathrm{K}-\mathrm{NN}$ algorithm is an algorithm known as nonparametric sample-based learning. The model in this algorithm memorizes the training data set and finds the nearest neighbors as many as $\mathrm{k}$ number given to the algorithm in the data set, depending on the distance metric selected, and determines the classification tag of the data according to the majority. The K-Nearest neighbor algorithm allows easy adaptation for new data. However, it should be kept in mind that the cost of calculating these new samples increases in the worst case in direct proportion to the number of samples in the training data set, which is one of the main disadvantages of this algorithm.

\subsection{Linear Discrimination Analysis Algorithm \\ (Doğrusal Ayrumcılık Analizi Algoritması)}

LDA is an unsupervised algorithm used to reduce the number of dimensions in the data set. LDA algorithms can also be used as a feature extraction technique to increase computational efficiency, and reduce overfitting due to "curse of dimension" in dirty data [20]. LDA computes the $\mathrm{N}$-dimensional mean vector for each class by standardizing an Ndimensional data set creates the distribution matrix between and within classes. Converts matrices created here into low dimensional feature spaces. It is an algorithm that uses the covariance matrix to parse the data set. It works according to Fisher's score function, and estimates the linear coefficients that maximize the score. It tries to find linear combinations of variables that best describe the data and tries to model the difference between data classes. It can be used as a pre-process to extract features and identify classes prior to classification operations. 


\subsection{Gaussian Naive Bayes Algorithm (Gaussian Nä̈ve Bayes Algoritması)}

GNB algorithm is one of the Naive Bayes algorithm types. GNB is the type of supervised learning algorithm used when it has properties consisting of continuous values. In this algorithm developed on the basis of Bayes' theorem, each feature is considered independent from each other, which allows it to perform better than dependent models such as logistic regression. Good results can be obtained in both small size data, and high size data. Before using the Naive Bayes algorithm, the preprocessing and feature selection of the data should be done very well. Because there are not many parameters that can be optimized to change the result in the Naive Bayes algorithm, this optimization should be done first.

\subsection{Logistic Regression Algorithm (Lojistik Regresyon Algoritmasi)}

LR algorithm is a supervised machine learning algorithm that is very easy to implement. It is used in regression problems as well as classification problems. It performs better in a classification that can be separated linearly. Logistic regression, which is a simple but powerful algorithm for linear and binary classification problems, gives better results especially when the variable type to be predicted is category type. The logistic regression algorithm establishes the relationship between the probability of a variable and its attributes in the data set. It deals not only with the class to be predicted but also with predicting the probability of the variable in the class. The algorithm uses the logit function to estimate the logarithmic rate of an event. The logit function takes input values between 0 and 1 and converts these data to values across the entire range of real numbers to represent a relationship.

\subsection{Random Forest Algorithm (Rassal Orman Algoritmast)}

$\mathrm{RF}$ is an algorithm used in both classification and regression problems that can prevent overfitting by creating more than one decision tree. Regardless of whether the data is separated linearly in the random forest algorithm, the most important features are inferred with all the decision trees created. Unlike the classical decision tree algorithm, the random forest algorithm tries to find the most important one in a random feature subset instead of finding the most important feature when dividing a node into subnodes.

\section{RESULTS (SONUÇLAR)}

Before performing image transformation over time series with the sonar signals in the data set, classical classification algorithms such as; DT, K-NN, LDA, Gaussian NB, LR, RF, and SVM algorithms have been trained and tested for this problem. Each algorithm grid-based hyper parameter search method was applied, and results were noted for the reserved and unseen test set. In order to identify the features for the classical machine learning algorithms, energy levels obtained from 60 frequency bands were used as features in the data set. For classical machine algorithms, feature selection or feature engineering methods were not performed. A new dataset is created with the pictures obtained with image transformation over time series methods in the same order as the case of the classical algorithms. In both cases, $80 \%$ of this dataset was used for training and $20 \%$ for testing. All the algorithms' performance was measured with accuracy, precision, recall and F1 - Score metrics and given in Equations (13-16).

$$
\begin{aligned}
& \text { Accuracy }=\frac{\mathrm{TP}+\mathrm{TN}}{\mathrm{TP}+\mathrm{TN}+\mathrm{FP}+\mathrm{FN}} \\
& \text { Precision }=\frac{\mathrm{TP}}{\mathrm{TP}+\mathrm{FP}} \\
& \text { Recall }=\frac{\mathrm{TP}}{\mathrm{TP}+\mathrm{TN}} \\
& F_{1}-\text { Score }=\frac{2 \cdot \text { Precision } * \text { Recall }}{\text { Precision }+ \text { Recall }}
\end{aligned}
$$

In these equations, $T_{P}$ represents the number of the actual positive values, $T_{N}$, the true negative values, $F_{P}$ the false positive value and the $F_{N}$ the negative value. Using these values, precision, recall, accuracy and $F_{l}$ criterion values are calculated for each algorithm to measure their performances.

The radial based function and $\mathrm{C}=1.5$ parameter values, which are among the nonlinear SVM algorithm kernel methods, have been studied, and the highest success rate has been achieved. DT algorithm parameters used in this study and giving the best results; Gini Information Criterion, the minimum number of instances required to split a node is 2 , the minimum number of instances in a leaf node is 1 , the maximum leaf node is not used, the class weight is not used, and the complexity parameter is 0.0 . K-NN algorithm achieved the highest success rate when the value of $\mathrm{k}$ was trained as 1 with the data set in this work. Uniform value was given as weight, and auto was chosen as the algorithm, enabling it to decide the most appropriate algorithm according to the values. Since the data set in this study has many features, while working with the parameters of the LDA algorithm, the covariance matrix was not calculated using singular value decomposition (SVD) as a 
solvent. In addition to this parameter, the tolerance value was selected as 1.0e-4 because the other parameters were used as shrinkage auto, priority none, the number of components for dimension reduction none, covariance storage false and the solvent SVD. Random forest, parameters to achieve the best results with the data set in this study, the division criterion entropy, the maximum depth 15 , the logarithm of the function required to find the maximum number of features, the minimum number of samples in a leaf node is 5 , the minimum number of samples required to divide a node is 3 , The number of trees to be created was 1000, and the number of processors was unlimited.

All the results obtained from these algorithms are given in Table 2. Among the success rates, the highest result with $0.90 \%$ was SVM and the random forest algorithms with $0.86 \%$. Gaussian NB with the data set used in this work, the previous probabilities of the classes were not specified in the parameters studied in order to achieve the most ideal results, and the smoothing value required for calculation stability was chosen as 1.0e-9. Logistic regression, in this study, in order to achieve the most ideal results with the data set, the parameters were chosen as penalty norm L2, the solver as "linear" because the size of the data set was not high, the maximum iteration number was 100 , and the warm start value was chosen not to be used because the solver value was linear. Table 3 shows the results obtained from the $\mathrm{CNN}$ architecture with the time series image transformation method for the ICENTE20 conference [21]. The results of each transformation method are stated separately in the study conducted at the conference.

Among the deep learning techniques, a convolutional neural networks architecture, which is quite successful and popular in image processing, was established, trained, and tested. The proposed custom convolutional neural network architecture is trained separately for each transformation process after data transformations are completed. Binary cross entropy loss function with L2 regularization is used for optimization and for training, Adam optimization algorithm with learning rate $1.0 \mathrm{e}-4$ was chosen and iterated over 15 epochs. Throughout the iteration, the learning rate has been decreased to a certain extent and it has been tried to reach the minimum value in the loss function and 5-fold cross validation was applied. Accuracy, precision, recall, and F1 measure values obtained with this convolutional neural network model trained after image fusion and merging operations for extra information method was $97.60 \%$, $\% 98.01, \% 97.20$, and \%97.60, respectively. The results and metrics obtained with this proposed method are given in Table- 4 .
Table 1. Comparison of results obtained from studies in literature review (Literatürde incelenen çalışmaların sonuçlarının karșılaștırılması)

\begin{tabular}{|c|c|c|}
\hline & Approach & Accuracy \\
\hline [10] & $\begin{array}{c}\text { Nearest Neighbor } \\
\text { Classification - 12 Hidden } \\
\text { Layer }\end{array}$ & 90.40 \\
\hline [11] & GRNN with PCA & 93.26 \\
\hline$[12]$ & RS1 & 49.50 \\
\cline { 2 - 3 } & RS2 & 51.95 \\
\cline { 2 - 3 } & RSU & 51.40 \\
\cline { 2 - 3 } & TS1 & 51.93 \\
\cline { 2 - 3 } & TS2 & 53.43 \\
\cline { 2 - 3 } & TSU & 49.98 \\
\cline { 2 - 3 } & RW1 & 51.93 \\
\cline { 2 - 3 } & RW2 & 52.88 \\
\hline [13] & RWU & 51.38 \\
\hline C14] & NBup with iDCM-CA & 81.00 \\
\cline { 2 - 3 } & SVM with iDCM-CA & 82.50 \\
\cline { 2 - 3 } & NN with iDCM-CA & 60.00 \\
\cline { 2 - 3 } & DT with iDCM-CA & 98.75 \\
\cline { 2 - 3 } & IBK with iDCM-CA & 86.25 \\
\cline { 2 - 3 } & LWL with iDCM-CA & 96.00 \\
\hline [15] & IMGMP & 82.79 \\
\hline$[16]$ & MIME-KNN & 82.20 \\
\hline
\end{tabular}

Table 2. Classical machine learning algorithms results of sonar signals in the data set with classification algorithms (Sinfflandırma algoritmalart ile veri setindeki sonar sinyallerinin klasik makine ögrrenmesi algoritmaları sonuçları)

\begin{tabular}{|c|c|c|c|c|c|}
\hline $\begin{array}{c}\text { ML } \\
\text { Algorithm }\end{array}$ & & Acc. & Prec. & Rec. & $\mathrm{F}_{1}$ \\
\hline \multirow[t]{2}{*}{ SVM } & $\mathrm{M}$ & \multirow[t]{2}{*}{0.90} & 0.88 & 0.95 & 0.91 \\
\hline & $\mathrm{R}$ & & 0.94 & 0.85 & 0.89 \\
\hline \multirow{2}{*}{$\begin{array}{l}\text { Random } \\
\text { Forest }\end{array}$} & $M$ & \multirow[t]{2}{*}{0.86} & 0.81 & 0.95 & 0.88 \\
\hline & $\mathrm{R}$ & & 0.94 & 0.75 & 0.83 \\
\hline \multirow{2}{*}{$\begin{array}{c}\text { Logistic } \\
\text { Regression }\end{array}$} & $M$ & \multirow[t]{2}{*}{0.83} & 0.80 & 0.91 & 0.85 \\
\hline & $\mathrm{R}$ & & 0.88 & 0.75 & 0.81 \\
\hline \multirow{2}{*}{$\begin{array}{c}\text { Gaussian } \\
\text { Naive Bayes }\end{array}$} & $\mathrm{M}$ & \multirow[t]{2}{*}{0.67} & 0.83 & 0.45 & 0.59 \\
\hline & $\mathrm{R}$ & & 0.60 & 0.90 & 0.72 \\
\hline \multirow[t]{2}{*}{ Decision Tree } & $\mathrm{M}$ & \multirow[t]{2}{*}{0.71} & 0.75 & 0.68 & 0.71 \\
\hline & $\mathrm{R}$ & & 0.68 & 0.75 & 0.71 \\
\hline \multirow[t]{2}{*}{ K-NN } & $\mathrm{M}$ & \multirow[t]{2}{*}{0.86} & 0.83 & 0.91 & 0.87 \\
\hline & $\mathrm{R}$ & & 0.89 & 0.80 & 0.84 \\
\hline \multirow[t]{2}{*}{ LDA } & M & \multirow[t]{2}{*}{0.76} & 0.77 & 0.77 & 0.77 \\
\hline & $\mathrm{R}$ & & 0.75 & 0.75 & 0.75 \\
\hline
\end{tabular}

Table 3. Time series - Image transformation method and the best results of the proposed custom CNN architecture at the ICENTE20 conference. [21] (ICENTE20 konferansinda yapılmuş olan zaman serisi - görüntü dönüşümü yöntemi ile önerilen CNN mimarisine ait elde edilen en iyi sonuçlar

\begin{tabular}{|c|c|c|c|c|}
\hline & Accuracy & Precision & Recall & $\mathrm{F}_{1}$ \\
\hline RP & 0.9434 & 0.9340 & 0.9620 & 0.9477 \\
\hline MTF & 0.9323 & 0.9565 & 0.9212 & 0.9385 \\
\hline GADF & 0.9057 & 0.9167 & 0.8798 & 0.8979 \\
\hline
\end{tabular}


Table 4. Time-series image transformation with image merging method and the best result of the proposed custom $\mathrm{CNN}$ architecture (Zaman serisi görüntü dönüşümü yöntemi ile önerilen CNN mimarisinden elde edilen en iyi sоnuç)

\begin{tabular}{|c|c|c|c|}
\hline Accuracy & Precision & Recall & $\mathrm{F}_{1}$ \\
\hline 0.9760 & 0.9801 & 0.9720 & 0.9760 \\
\hline
\end{tabular}

\section{CONCLUSION (SONUÇ)}

This study proposed a novel information representation and fusion method to obtain higher performance for time-series classification problems without using any feature engineering and feature selection methods. The proposed method consists of converting regular time series to an image representation by implementing MTF, RP, and GAF transformations. In the final stage, separate data from the transformation merged as RGB channels to form an ultimate image were used. It is seen that the proposed method can be used as a more efficient feature extractor than classical methods thanks to RP, GAF, and MTF transformations.

By comparing with our previous studies [21], the performance of classical ML methods and DL based time series-image transformation methods, it was seen that the proposed method in this work could reach the best accuracy, precision, recall, and F1 score values. From Table 2, it was determined that the counting based probabilistic image transforms give better results for this data set. It is thought that the proposed method can be used as a more efficient feature extractor than classical methods thanks to RP, GAF, and MTF transformations, and it is the transformation method that takes into account the spatial correlation. When the approaches and methods in the literature are reviewed, it is seen that the methods in which size reduction approaches are used to give more successful results.

These dimension reduction algorithms and other feature extraction methods used in the preprocessing phase are used in the solution of the binary classification problem and utilized as input for machine learning and statistical pattern recognition methods. Our results prove that usage of the computer vision-based DL algorithms without doing any feature engineering or selection can be proper for classification problems, especially for time series data. It was discovered that the method proposed in this study gave the best results reported in the literature for the sonar and mine classification problem specific to the sonar dataset.

\section{NOTE (NOT)}

This study was expanded from the paper published at the International Conference and Engineering Technologies (ICENTE20) conference and was first published in the ICENTE20 Proceedings Book on 28 December 2020. ICENTE20 conference was held in Konya on 19-21 November 2020.

\section{CONFLICT OF INTEREST STATEMENT (ÇIKAR ÇATISSMASI BILDIRIMI)}

The authors reported no potential conflict of interest.

\section{REFERENCES (KAYNAKLAR)}

[1] A.D. Waite Sonar for Practising Engineers. Wiley, West Sussex, England, 2002.

[2] L. Jing, "The principle of side scan sonar and its application in the detection of suspended submarine pipeline treatment." Materials Science and Engineering, vol. 439, no.3, pp. 032068, 2018. doi: 10.1088/1757-899X/439/3/032068

[3] V.L. Lucieer. "Object-oriented classification of sidescan sonar data for mapping benthic marine habitats," International Journal of Remote Sensing, vol. 29, no. 3, pp. 905-921, 2018. doi: 10.1080/01431160701311309.

[4] A. Burguera, G. Oliver, "High-Resolution Underwater Mapping Using Side-Scan Sonar." PLOS ONE, vol. 11, no.1, 2016. doi: 10.1371/journal.pone. 0146396 .

[5] H.J. Flowers, J.E. Hightower, "A Novel Approach to Surveying Sturgeon Using Side-Scan Sonar and Occupancy Modeling." Marine and Coastal Fisheries, vol. 5, no.1, pp. 211-223, 2013. doi: $10.1080 / 19425120.2013 .816396$

[6] A.T. Çelebi, M.K. Güllü, S. Ertürk, "Mine detection in side scan sonar images using Markov Random Fields with brightness compensation," in 2011 IEEE 19th Signal Processing and Communications Applications Conference (SIU), 2022 April 2011, Antalya, Turkey, 2011, pp. 916-919.

[7] C.S. Huebner, "Evaluation of side-scan sonar performance for the detection of naval mines." Target and Background Signatures IV, vol. 10794, pp. 158166, 2018. doi: https://doi.org/10.1117/12.2325642

[8] M. Verleysen, D. François, "The Curse of Dimensionality in Data Mining and Time Series Prediction." Lecture Notes in Computer Science, vol. 
3512, no.6, pp. 758-770, 2005. doi: 10.1007/11494669_93

[9] R.P. Gorman, T.J. Sejnowski, “Analysis of Hidden Units in a Layered Network Trained to Classify Sonar Targets." Neural Networks, vol. 1, no.1, p p . 75-89, 1988. doi: https://doi.org/10.1016/08936080(88)90023-8

[10] B. Erkmen, T. Yıldırım. "Improving classification performance of sonar targets by applying general regression neural network with PCA." Expert Systems with Applications,. vol. 35, no.1-2, pp. 472-475, 2008. doi: https://doi.org/10.1016/j.eswa.2007.07.021

[11] M. Hossin, F. Mahudin, I. Din, A.R. Mat. "Analysis of Nine Instance-Based Genetic Algorithm Classifiers Using Small Datasets." Journal of Telecommunication, Electronic and Computer Engineering, vol. 9, pp 3-11, 2017.

[12] J. Novakovic. "Using Information Gain Attribute Evaluation to Classify Sonar Targets," in 17th Telecommunications forum TELFOR 2009. 24-26 November 2009. Serbia, Belgrade.

[13] S. Fong, S. Deb, R. Wong, et al. "Underwater Sonar Signals Recognition by Incremental Data Stream Mining with Conflict Analysis," International Journal of Distributed Sensor Networks, vol. 10, no.5, pp 1-12 May 2014. doi: 10.1155/2014/635834

[14] X. Hong, J. Zhang, S.U. Guan. "Incremental Maximum Gaussian Mixture Partition For Classification," in 2nd Joint International Information Technology, Mechanical and Electronic Engineering Conference (JIMEC 2017), 2017, pp. 62.

[15] T. Shang, X. Xia, J. Zheng. "MIME-KNN: Improve KNN Classifier Performance Include Classification Accuracy and Time Consumption." DEStech Transactions on Computer Science and Engineering, July $2018 . \quad$ doi: 10.12783/dtcse/csse2018/24490

[16] Z. Wang, T. Oates. "Imaging Time-Series to Improve Classification and Imputation." Proceedings of the Twenty-Fourth International Joint Conference on Artificial Intelligence. (IJCAI), 2015.

[17] Z. Wang, T. Oates. "Spatially Encoding Temporal Correlations to Classify Temporal Data Using Convolutional Neural Networks." Journal of Computer and Systems Sciences, 2015.

[18] J.P. Eckmann, S.O. Kamphorst, D. Ruelle "Recurrence Plots of Dynamical Systems." Euro-
Physics Letters (EPL). vol. 4, no. 9, pp. 973-977, 1987.

[19] A. Şeker, B. Diri, H.H. Balık. "Derin Öğrenme Yöntemleri ve Uygulamaları Hakkında Bir İnceleme." Gazi Mühendislik Bilimleri Dergisi, vol. 3, no. 3, pp. 47-64, 2017.

[20] S. Raschka, V. Mirjalili. Python Machine Learning, 2nd Edition 2017.

[21] A. Civrizoğlu Buz, M.U. Demirezen, U. Yavanoğlu. "A Novel Approach and Application of Time Series to Image Transformation Methods on Classification of Underwater Objects," in Proceedings of the 4th International Conference on Engineering Technologies (ICENTE'20). Konya, Turkey. November 19-21, 2020, pp. 31-37 [Online]. 